\title{
Unsteady Aerodynamics of Flapping Wing of a Bird
}

\author{
M. Agoes Moelyadi ${ }^{1}$, Hendra Adi Putra ${ }^{1}$ \& Gottfried Sachs ${ }^{2}$ \\ ${ }^{1}$ Faculty of Mechanical and Aerospace Engineering, Bandung Institute of Technology, \\ Jalan Ganesa No. 10, Bandung 40132, Indonesia \\ ${ }^{2}$ Institute of Flight System Dynamics, Technische Universität München, \\ Boltzmannstrasse 15, Garchingbei München, D-85748, Germany \\ Email: moelyadi@ae.itb.ac.id
}

\begin{abstract}
The unsteady flow behavior and time-dependent aerodynamic characteristics of the flapping motion of a bird's wing were investigated using a computational method. During flapping, aerodynamic interactions between bird wing surfaces and surrounding flow may occur, generating local time-dependent flow changes in the flow field and aerodynamic load of birds. To study the effect of flapping speed on unsteady aerodynamic load, two kinds of computational simulations were carried out, namely a quasi-steady and an unsteady simulation. To mimic the movement of the down-stroke and the upstroke of a bird, the flapping path accorded to a sinus function, with the wing attitude changing in dihedral angle and time. The computations of time-dependent viscous flow were based on the solution of the Reynolds Averaged Navier-Stokes equations by applying the $\mathrm{k}-\varepsilon$ turbulence model. In addition, the discretization for the computational domain around the model used multi-block structured grid to provide more accuracy in capturing viscous flow, especially in the vicinity of the wing and body surfaces, to obtain a proper wing-body geometry model. For this research, the seagull bird was chosen, which has high aspect ratio wings with pointed wing-tips and a high camber wing section. The results include mesh movement, velocity contours as well as aerodynamic coefficients of the flapping motion of the bird at various flapping frequencies.
\end{abstract}

Keywords: dynamic grids; flapping wing motion; flapping frequency; seagull bird; unsteady aerodynamics.

\section{$1 \quad$ Introduction}

Research on the aerodynamics of bird flight still receives attention from many researchers. Indeed, interest has increased considerably due to a growing interest in developing micro aerial vehicles (MAVs). Most birds produce lift and thrust by flapping their wings for flight. As the flapping motion occurs, the wings passively deform during the down-stroke, creating a nose-down cambered wing and generating more thrust and lift. During the upstroke, the trailing edge of the outer part of the flexible wing deforms strongly to reduce drag, but less lift is produced. The resulted Local time-dependent flow during the flapping motion has an important effect on generating thrust and lift by the

Received August $16^{\text {th }}, 2011,1^{\text {st }}$ RevisionMay $16^{\text {th }}, 2012,2^{\text {nd }}$ Revision August $31^{\text {st }}, 2012,3^{\text {rd }}$ Revision January $26^{\text {th }}, 2013$, Accepted for publication January $28^{\text {th }}, 2013$.

Copyright @ 2013 Published by ITB Journal Publisher, ISSN: 2337-5779, DOI: 10.5614/j.eng.technol.sci.2013.45.1.4 
bird wing. Research on the unsteady aerodynamics of the flapping motion has been done by several researchers. Jones and Platzer [1] made their contribution by calculating the in viscid flow for two-dimensional airfoils in plunging and pitching motion in comparison with analytical results. Moelyadi and Sach [2] have simulated flapping motion with a small dihedral angle for calculating dynamic stability derivatives in the yaw for a bird. Also, aerodynamic effects on birds with a large dihedral angle have been studied [3]. Navier-Stokes solutions for the viscous flow around two-dimensional wings have been presented by Isogai [4]. Persson, et al. have carried out a numerical simulation of a flapping wing at Low Reynolds numbers for an elliptical wing planform with the flow solution obtained by solving the Navier-Stokes equations [5]. Mantia and Dabnichki [6] studied the effect of wing shape on the thrust of a flapping wing by simulating a harmonically oscillating wing using the boundary element method. Many researchers still use simple geometry for their computational models.

This paper presents the effects of the flapping motion of a bird wing on unsteady flow behavior and time-dependent aerodynamic characteristics of the complete wing and body. The seagull bird was chosen as a case study, which has high aspect ratio wings with pointed wing-tips and a high camber wing section. The simulations were conducted for three different flapping frequencies, namely 3,6 and $12 \mathrm{sec}^{-1}$. To mimic the down-stroke and upstroke movement of the bird, the wing position changed in dihedral angles with respect to time and the path motion according to a sinus function. The unsteady and viscous flows at low speed were evaluated using the solution of time-dependent Reynolds Averaged Navier-Stokes equations with the k- $\varepsilon$ turbulence model. In addition, the space discretization for the computational domain around the bird model used a multi-block structured grid technique to provide more accuracy in capturing viscous flow, especially in the vicinity of the wing and body surfaces, and to properly model the wing-body bird complex.

\section{Computational Simulation of Flapping Bird}

\subsection{Motion Modeling}

Aerodynamic forces exerted on the bird due to the flapping motion are assumed to be a function of amplitude with respect to time as well as the frequency of the flapping wing. In the simulation, the motion path of the flapping wing was written as follows:

$$
\Psi(\mathrm{t})=\Psi \max \sin (2 \pi f \mathrm{t})
$$

where, $\Psi$ is amplitude, $\mathrm{t}$ is time, and $f$ is flapping frequency. Most bird's wings not only have flapping movement, but there are also other movements, like 
twisting (feathering), lagging, and spanning [7]. In this research the flapping motion of the wing was simplified by considering only the changes of vertical motion in dihedral angles, with no twisting (feathering). In addition, it was assumed that the wing is rigid during the flapping motion.

\subsection{Geometry, Topology and Mesh Generation}

Before the computation of the flow can be executed, the definition of the computational domain, including the creation of the bird's geometry and mesh generation, has to be carried out first. The mesh generated in the computational domain has to accurately simulate flow physics, such as viscous flow, and to properly model the wing body bird complex. It is important to obtain a high quality (orthogonal) mesh. For complex geometries such as the body and wings of a bird, atopology with a multi-block structured grid is required.

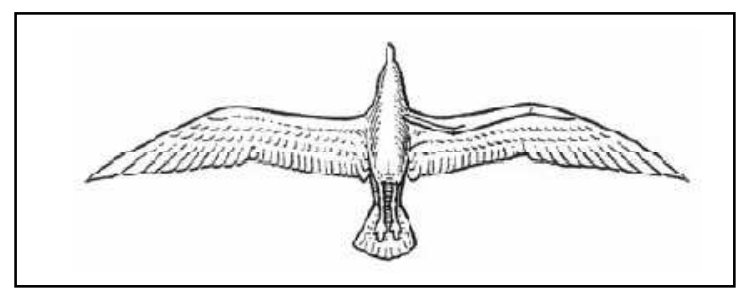

Figure 1 Seagull bird (from ref. [8]).

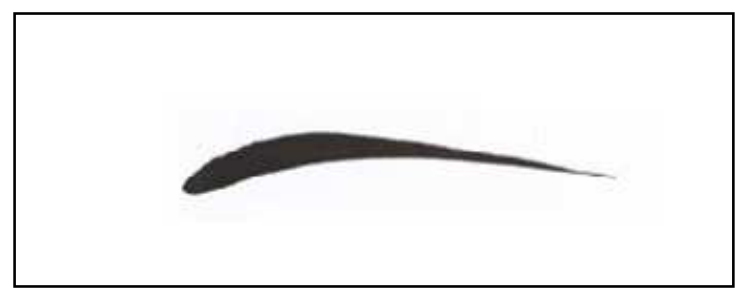

Figure 2 Wing section of seagull bird (from ref. [8]).

The seagull's configuration is depicted in Figure 1, and its wing profile in Figure 2. The data of the seagull are the following [8]:

$\begin{array}{ll}\text { Area reference: } & 0.188 \mathrm{~m}^{2} \\ \text { Length reference: } & 0.160 \mathrm{~m} \\ \text { Span reference: } & 1.560 \mathrm{~m} \\ \text { Total length: } & 0.650 \mathrm{~m} \\ \text { Mass: } & 1.607 \mathrm{~kg} \\ \text { Minimum speed: } & 11.7 \mathrm{~m} \cdot \mathrm{s}^{-1} \\ \text { Maximum speed: } & 20.6 \mathrm{~m} \cdot \mathrm{s}^{-1}\end{array}$


The geometry of the wing-body complex of the seagull bird was generated directly using the ICEM CFD meshing software. The wing planform was formed from several wing sections which were arranged along the wing span as shown in Figure 3.

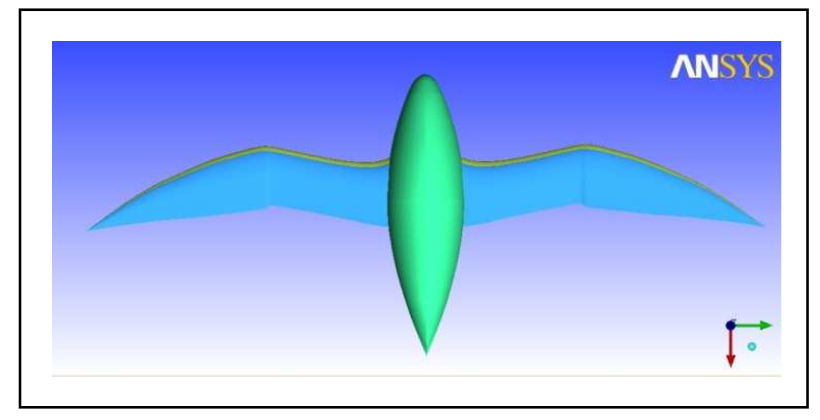

Figure 3 Computational model of seagull bird.

\subsubsection{Topology of Seagull bird}

Having created the geometry of the wing-body complex of the seagull, the block topology model was generated on the underlying geometry. The computational domain was divided into several smaller block domains using a multi-block technique with the intention of obtaining a high-quality grid and hence accurate solutions. In order to obtain a more realistic solution, the definition of the boundaries, including far-field, inlet, outlet and solid boundary, was accomplished. For example, the far-field boundary was defined as the outer boundary of the computational domain with a distance of about 7 to 10 times of the bird's length. The solid boundary was applied to the geometry of the body and wings. The geometry of the bird was placed in the DEAD block. The blocks around the bird used for computation were labeled as LIVE block. Because the simulation case was symmetry flow, the topology was created for a half configuration in order to reduce computation time. The total number of blocks used for the half configuration of the bird was 26 blocks. The block topologies are shown in Figure 4.

\subsubsection{Mesh Generation}

After the block topology was formed, the points for each block were distributed along the edge of the block. It is necessary to concentrate a major number of points in critical regions, such as the leading-edge and trailing-edge sections of the wing. Point distribution in the computational domain becomes denser when the points approach the body surface. In order to capture viscous flow on the wing surface and body more correctly, it was necessary to determine the 
distance of the grid closest to the surface by determining the value of $y+$. Based on a Reynolds number of about $0.4 \times 10^{6}, y+=1$ and the reference length of 0.16 $\mathrm{m}$, the distance was $0.337 \mathrm{~mm}$. The mesh generation of the seagull wing with a total number of cells of about $3.6 \times 10^{5}$ is shown in Figure 5 .

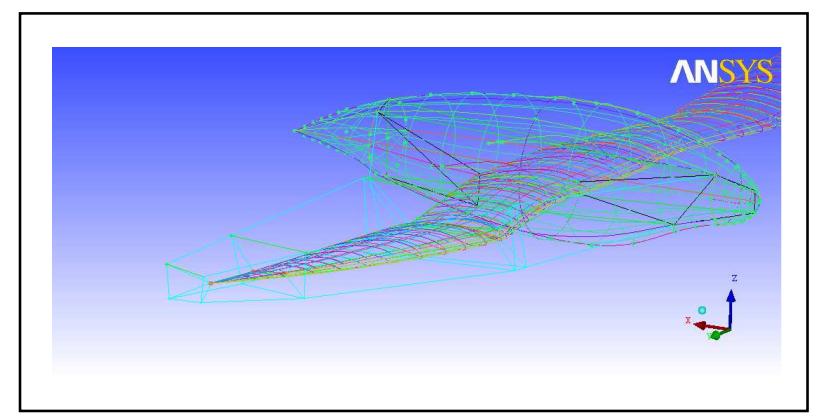

Figure 4 Topology around seagull wing.

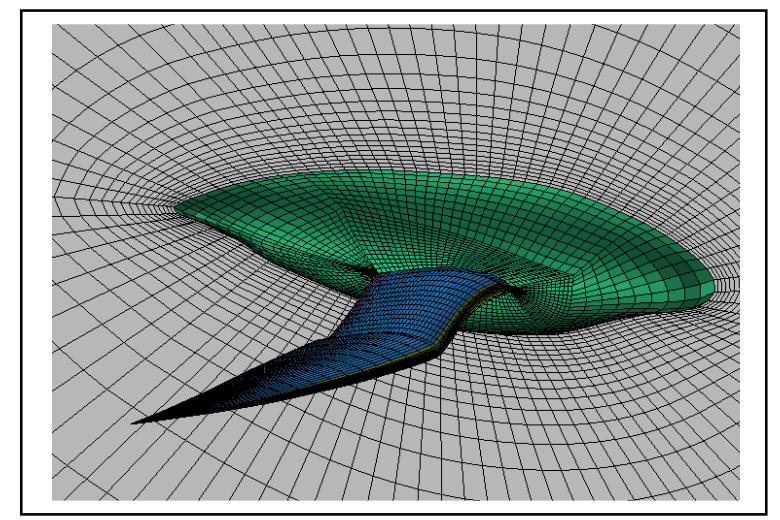

Figure 5 Mesh generation for seagull wing.

\subsection{Numerical Simulation Method}

Both the computation of unsteady flow and quasi-steady flow was obtained using the ANSYS CFX software application. The Reynolds-averaged NavierStokes equations were solved with the $\mathrm{k}-\varepsilon$ turbulence model. This turbulence model can capture flow dominated by large eddies more accurately.

\subsubsection{Quasi-steady Simulation}

The quasi-steady simulations were performed by having the wings on the body moving from a lower position to a higher position without considering the effect of local time-dependent flow. The quasi-steady simulations were carried out for five different dihedral angles, namely $-10,-5.0,0.0,5.0$, and 10 degrees. 


\subsubsection{Unsteady Simulation}

The unsteady simulations were carried out by flapping the bird wing periodically with a given flapping frequency. The grid blocks in the computational domain around the model moved to follow the wing's movement. The wing's movement path was stated as:

$$
\Psi(\mathrm{t})=\tan \left(10^{\circ}\right)(\mathrm{y}-0.0844 \mathrm{~m}) \sin (2 \pi f \mathrm{t})
$$

The maximum dihedral angle was $10 \mathrm{deg}$. The rotation based on the $\mathrm{x}$-axis located at $\mathrm{y}$ was equal to $0.0844 \mathrm{~m}$. The results of the moving grid blocks for snap shots of the flapping motion at various dihedral angles are shown in Figure 6.

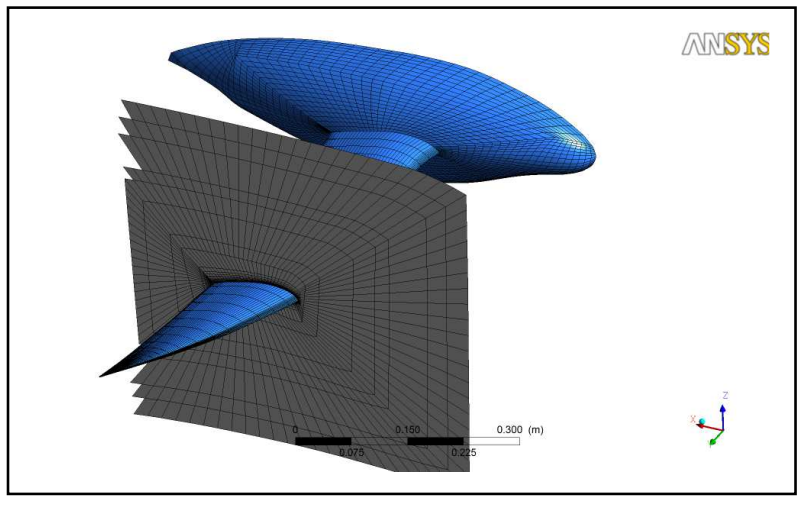

(a) $2 \mathrm{y} / \mathrm{b}=0.78$ and $\psi=0$ deg.

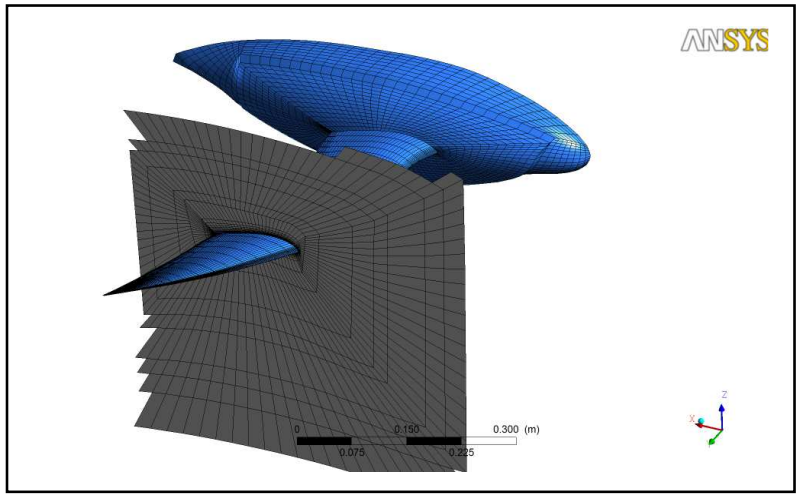

(b) $2 \mathrm{y} / \mathrm{b}=0.78$ and $\psi=10 \mathrm{deg}$.

Figure 6 Dynamic grid for flapping motion of seagull wing. 


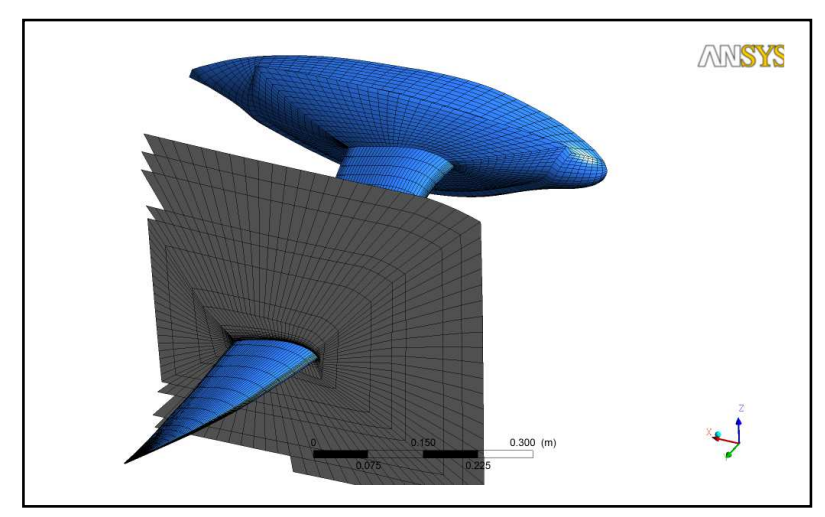

(c) $2 \mathrm{y} / \mathrm{b}=0.78$ and $\psi=-10 \mathrm{deg}$.

Figure 6 Continued. Dynamic grid for flapping motion of seagull wing.

In the unsteady simulation, three flapping frequencies were computed, namely $f$ $=3,6$ and $12 \mathrm{~s}^{-1}$. The unsteady calculations started with the use of the input of the steady state solution. Subsequently, the unsteady solution for each time step was obtained by solving the unsteady Navier-Stokes equations using the dual time-stepping method. Every time-step the body changes its position, inducing mesh deformation near the wing's surface. This required a dynamic grid adaption, generated by local grid-smoothing using the Laplace algorithm.

In order to obtain accurate computation results, the computations were carried out for three cycles with one cycle consisting of 40 time-steps, that is, from the initial position (middle) and back to the initial position again. The convergence solution for each time-step of the simulation was yielded when the residual of relative density reached a value of $1 \times 10^{-5}$.

\section{$3 \quad$ Results and Discussion}

\subsection{Aerodynamic Characteristics}

Figures 7-9 show the results of the third cycle of the unsteady aerodynamic characteristics of the flapping wing. The free stream condition was set at a velocity of $12.5 \mathrm{~m} / \mathrm{s}$. For comparison, the results of the quasi steady simulation are also given, showing that the effect of the dihedral angle gave slight changes in lift and drag coefficients, while the magnitude of the pitching moment slightly decreased withan increase in dihedral angle.

Compared to the quasi-steady simulation results, the unsteady simulation results give an amount of additional lift coefficient during down-stroke. The maximum increment of the additional lift was obtained when the wing reached the middle 
position. This is caused by the higher local time-dependent flow velocity occurring at the middle position of the wing. During upstroke the lift coefficient continuously decreased. The maximum decrement of the lift coefficient occurred at the normal/middle wing position. At this position the wing still provides a positive lift, even if the flight level of the bird may slightly decrease. Furthermore, increasing the flapping frequency gave a larger amount of additional lift coefficient during down-stroke at the middle wing position. However, during upstroke at a higher flapping frequency, a large reduction of the lift coefficient caused negative lift coefficient of the wing. As a result, the wing may experience changes in vertical position during flapping in flight. The simulation results gave the optimum flapping frequency for the seagull bird as $3 \mathrm{~s}^{-1}$, the same as stated in reference [8].

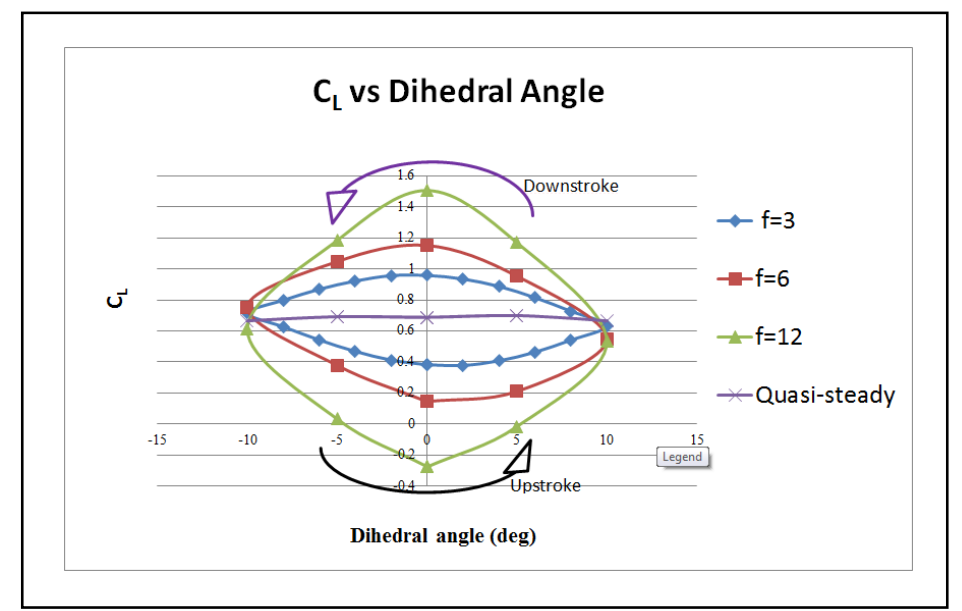

Figure 7 Lift coefficient of seagull bird at various flapping frequencies.

Concerning the drag coefficient as shown in Figure 8, the flapping motion during upstroke gave a slight increase in drag coefficient. During down-stroke, the wing gave a reduction in drag coefficient. In addition, at a flapping frequency of $3 \mathrm{~s}^{-1}$ the wings produced a small positive drag coefficient. However, higher flapping frequencies yielded negative drag or thrust during down-stroke. To produce thrust at a lower flapping frequency, a twisting motion combined with the flapping motion during down-stroke may be considered.

Concerning the pitching moment coefficient as shown in Figure 9, the flapping motion of the bird provided a certain amount of more negative pitching moment during down-stroke and a certain amount of less negative pitching moment compared to the results of the quasi-steady simulation. At a flapping frequency of $3 \mathrm{~s}^{-1}$ the differences in pitching moment coefficient between the downstroking and up-stroking were small. However, at the higher flapping 
frequencies the differences in pitching moment coefficient between the downstroking and the up-stroking were large. The differences in pitching moment increased with an increase in flapping frequency. This indicates that the seagull is more stable in flight at a flapping frequency of $3 \mathrm{~s}^{-1}$, with less changes in pitching moment.

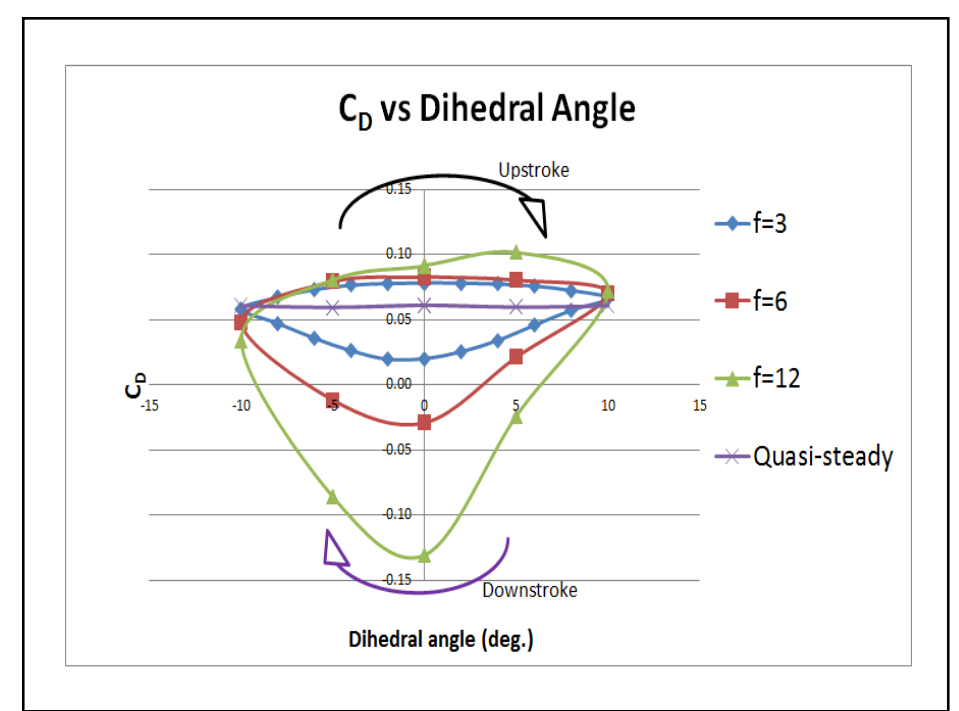

Figure 8 Drag coefficient of seagull bird at various flapping frequencies.

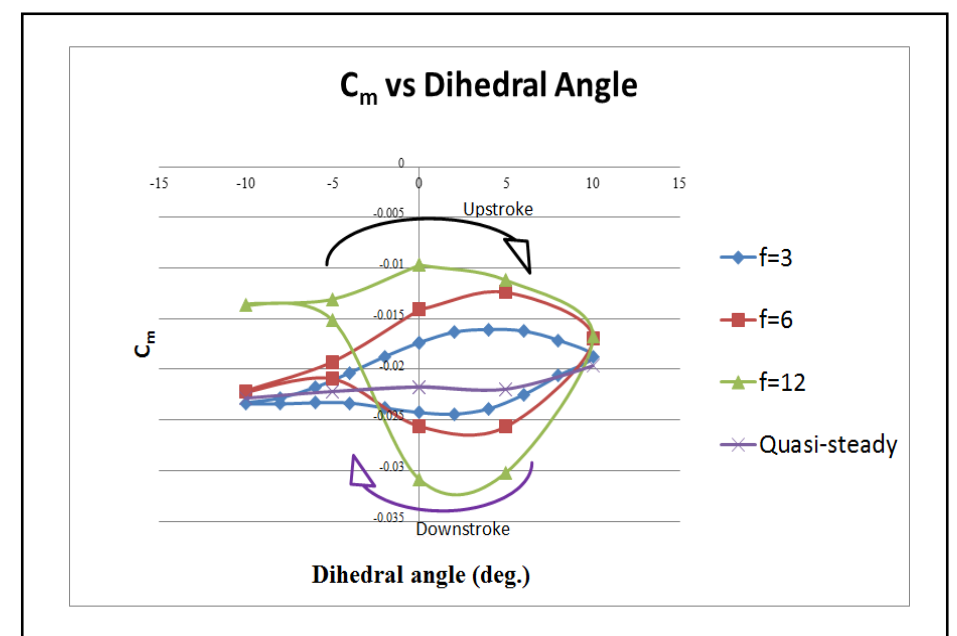

Figure 9 Pitching moment coefficient of seagull bird at various flapping frequencies. 


\subsection{Flow Behaviour}

Figures 10-12 show the velocity contours for the quasi-steady solution and the unsteady solution for down-stroke and upstroke at a location of the wing section of $2 y / b=0.78$. All compared plots of the velocity contours used the same lower and upper limits of velocity. Additionally, the analysis was also carried out when the wing was at the middle position, i.e. at a dihedral angle of zero. For the quasi-steady solution the flow accelerated from the leading edge of the wing to the wing's upper surface. The maximum velocity of about $19 \mathrm{~m} \cdot \mathrm{s}^{-1}$ occurred at $30 \%$ length of the wing section chord line.

Compared to the quasi-steady solution, the unsteady flow solution yielded a local time-dependent flow velocity during the down-stroke causing a change in the flow field around the wing section. At a flapping frequency of $3 \mathrm{~s}^{-1}$ the higher velocity region became larger on the upper surface, as shown in Figure 11(a). This yielded a lower pressure subjected to the upper surface and consequently an additional lift of the wing. Increasing the flapping frequency, the induction of the local time-dependent flow became larger, causing a higher increase in velocity of the flow on the upper surface, as shown in Figure11(b) and 11(c). The velocity on the upper surface can reach $24 \mathrm{~m} \cdot \mathrm{s}^{-1}$ at a flapping frequency of $12 \mathrm{~s}^{-1}$. The amount of lift due to the local time-dependent flow is more than twice the lift of the quasi-steady solution as shown in Figure 7. In addition, during down-stroke the rear part of the lower surface underwent a lower velocity or higher pressure load, resulting in more negative moment of the wing, as shown in Figure 9.

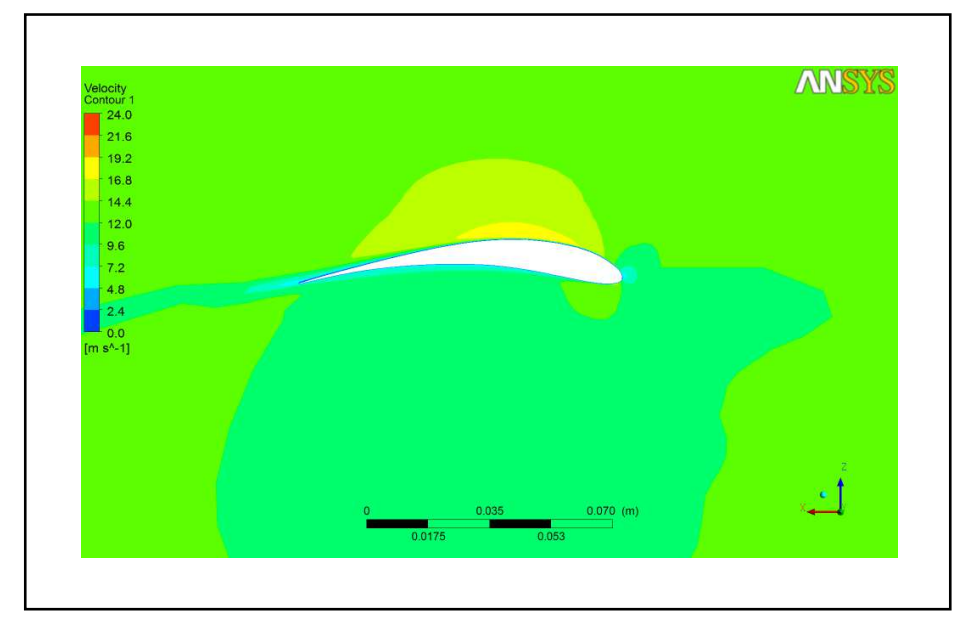

Figure 10 Velocity contour for quasi-steady condition at mid half wing span, $\mathrm{y} / 2 \mathrm{~b}=0.78$. 
Furthermore, the upstroke motion of the wing provided a downward local time dependent flow as shown in Figure 12. This is indicated by the increasing flow velocity on the lower surface. Based on Bernoulli's principle, the lower surface was subjected to lower pressure flow, so that the lift of the wing decreased. Increasing the flapping frequency, the region of higher velocity became larger on the lower surface, so that the lift of the wing decreased more and the pitching moment of the wing became less negative.

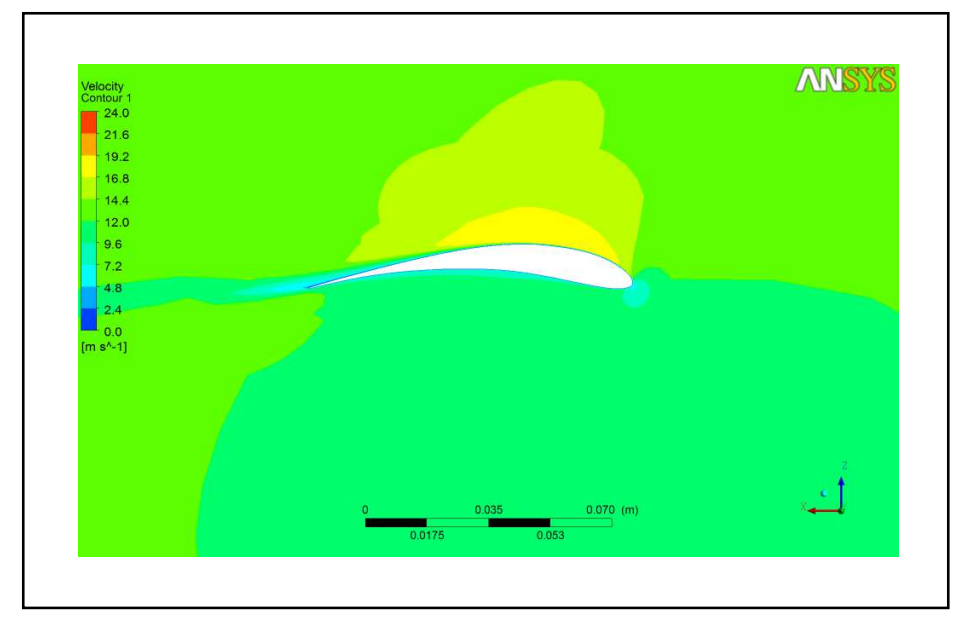

(a) Down-stroke ataflapping frequency of $3 \mathrm{sec}^{-1}$.

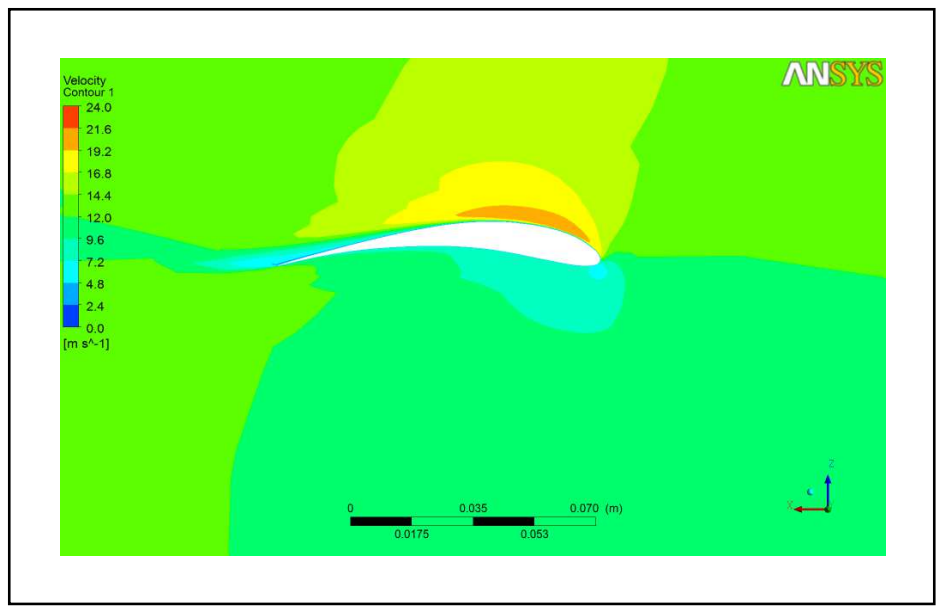

(b) Down-stroke at a flapping frequency of $6 \mathrm{sec}^{-1}$.

Figure 11 Velocity contour of unsteady flow condition of down-stroke at mid half wing span, $2 \mathrm{y} / \mathrm{b}=0.78$ at various flapping frequencies. 


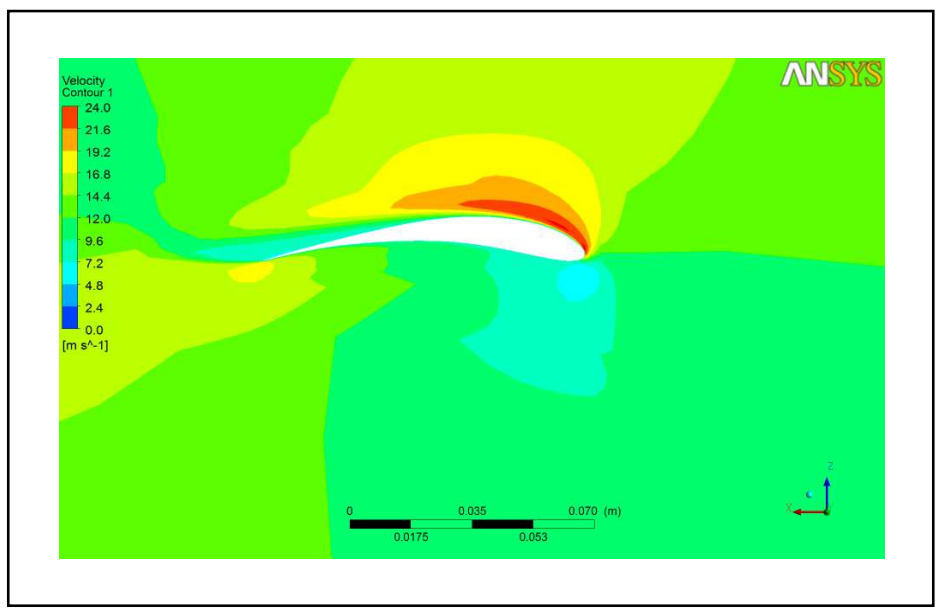

(c) Down-stroke at a flapping frequency of $12 \mathrm{sec}^{-1}$.

Figure 11 Continued. Velocity contour of unsteady flow condition of downstroke at mid half wing span, $2 \mathrm{y} / \mathrm{b}=0.78$ at various flapping frequencies.

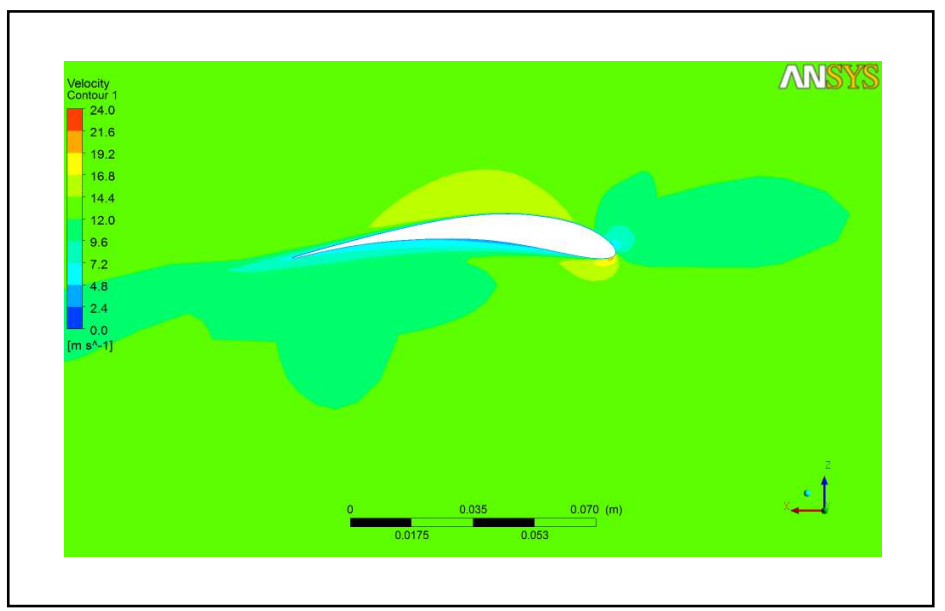

(a) Upstroke at a flapping frequency of $3 \mathrm{sec}^{-1}$.

Figure 12 Velocity contour of unsteady flow condition of upstroke at mid half wing span, $2 \mathrm{y} / \mathrm{b}=0.78$ at various flapping frequencies. 


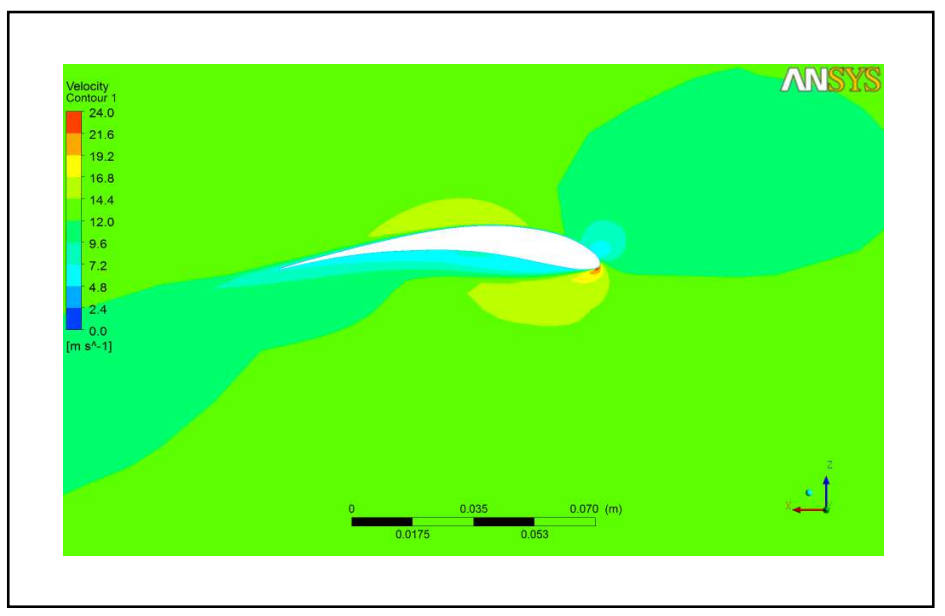

(b) Upstroke at a flapping frequency of $6 \mathrm{sec}^{-1}$.

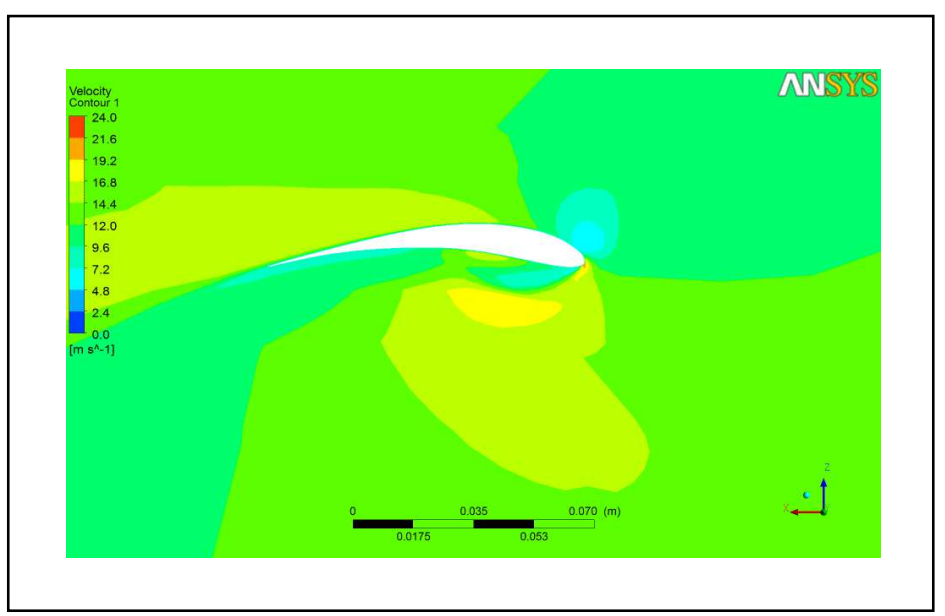

(c) Upstroke at a flapping frequency of $12 \mathrm{sec}^{-1}$.

Figure 12 Continued. Velocity contour of unsteady flow condition of upstroke at mid half wing span, $2 \mathrm{y} / \mathrm{b}=0.78$ at various flapping frequencies.

\section{Conclusions}

In this paper the simulation of unsteady flow around the flapping wing of a seagull bird was presented. The unsteady flow results, including time-dependent aerodynamic characteristics and unsteady flow behavior at upstroke and downstroke of the flapping wing, were compared with the corresponding quasi-steady results. The unsteady flow results show that during down-stroke the additional lift coefficient increased with increasing flapping frequency. In addition, thrust 
of the flapping wing was only obtained at higher flapping frequencies. During upstroke the amount of lift reduction increased with increasing flapping frequency. The greatest change in pitching moment occurred at a positive dihedral angle.

\section{Acknowledgement}

This research was a collaboration between the Institute of Technology Bandung, Indonesia and the Technische Universität München, Germany and was funded by the Ministry of Indonesian Education.

\section{References}

[1] Vest, M.S. \& Katz, J., Unsteady Aerodynamic Model of Flapping Wings, AIAA Journal, 34,(7), pp. 1435-1440, 1996.

[2] Moelyadi, M.A. \& Sachs, G., CFD Based Determination of Dynamic Stability Derivatives in Yaw for a Bird, Journal of Bionic Engineering, 4(4), pp. 201-208, 2007

[3] Sachs, G. \& Moelyadi, M.A., CFD Based Determination of Aerodynamic Effects on Birds with Extremely Large Dihedral, Journal of Bionic Engineering, 7(1), pp. 95-101, 2010.

[4] Isogai, K., Shinmoto, Y. \& Watanabe, Y., Effects of Dynamic Stall on Propulsive Efficiency and Thrust of Flapping Airfoil, AIAA Journal, 37(10), pp.1145-1151, 1999.

[5] Liu, H. \& Kawachi, K., A Numerical Study of Insect Flight, Journal of Computational Physics, 146(1), pp. 124-156, 1998.

[6] Mantia, M.L. \& Dabnichki, P., Effect of the Wing Shape on the Thrust of Flapping Wing, Applied Mathematical Modeling, 35, pp. 4979-4990, 2011.

[7] Michelson, R.C. \& Reece, S., Update on Flapping Wing Micro Air Vehicle Research, $13^{\text {th }}$ Bristol International RPV Conference, 1998.

[8] Herzog, K., Anatomy and Flight Biology of the Birds, Gustav Fischer Verlag, Stuttgart, Germany, 1968. 